\title{
Mobile Positioning Based on TOA/TSOA/TDOA Measurements with NLOS Error Reduction
}

\author{
Kuen-Tsiar Lay, and Wei-Kai Chao \\ Dept. of Electronic Engineering \\ National Taiwan Univ. of Science and Technology \\ Taipei, Taiwan, ROC \\ Tel: (816) 02-27376414, Fax: (816) 02-27376424 \\ Email: laykt@et.ntust.edu.tw
}

\begin{abstract}
With the spread of mobile communication networks, location dependent services are gaining popularity all over the world. Obviously, as its name implies, a location dependent service needs to know (more practically speaking, estimate) the location of the mobile station (MS) before it can offer any service at all. In this paper, we address the issue of mobile positioning (i.e. estimating the location of an MS) given the measured distances between the MS and its nearby base stations (BS's). The measurements, which are in general corrupted with measurement noise and NLOS (non-line-of-sight) error, can come from the time of arrival (TOA), the time sum of arrival (TSOA), or the time difference of arrival (TDOA). The NLOS error is the major cause that degrades the accuracy of mobile positioning. Assuming the knowledge of its statistic model, however, we propose a scheme that greatly reduce its effect. The results on MS location estimation thus obtained are comparable to the Cramer-Rao lower bounds (CRLB's). Regardless of the measurement types (i.e. TOA, TSOA, or TDOA), the proposed scheme computes the MS location in a unified way.
\end{abstract}

\section{INTRODUCTION}

With the rapid development of wireless communications, it is expected that location dependent services will be widely deployed all over the world in the near future. The first step in providing such services is, of course, to find the location of the client (i.e. MS) [1], [2]. It is quite probable that GPS (global positioning system) is the first thing popping up in our mind for this MS positioning task[3]. However, in this paper, we consider the situation that GPS is not available (e.g. inside a department store, where satellite signals are blocked by ceiling of building). Instead of using GPS, we assume that the positioning of the MS is to be based on its relative location with respect to nearby BS's, whose exact positions are already known.

Basically, we are going to measure the distances between the MS and the BS's (by TOA,TSOA, or TDOA) and then apply the method of trilateration to compute the MS's position. The problem is that the measured distances can be far from the true ones because of measurement noise and NLOS propagation error, in which the latter plays a dominant role in degrading the precision of the computed MS position[4]. Due to NLOS error, there tends to be an amount of overestimation in the measured distance. In this paper, by assuming a statistic model for the NLOS error, we are able to reduce this overestimation before trilateration is applied. Several types of models are considered. Algorithms for estimating the relevant parameters in those models are presented. Given the distributions of measurement noise (usually modeled as Gaussian) and NLOS error, the distribution of the measured distance can be derived. Then, the maximum likelihood estimate (MLE) of MS's location is computed. Due to the intractability of the measured distance's probability density function (pdf) in some cases, however, Gaussian pdf may have to be adopted

This work is partially supported by National Science Council under Grant No. 93-2213-E011-075. as an approximation. Moreover, with Gaussian approximation, we are able to derive a unified (taking into account of TOA, TSOA, and TDOA measurements) closed-form expression for the CRLB of the estimated MS location's variance. The CRLB will serve as a benchmark for evaluating the effectiveness of MS positioning schemes.

The rest of this paper is organized as follows. Sec. II describes the models and distributions of measured distances. The proposed MS positioning scheme is presented in Sec. III. In Sec. IV, experimental results are displayed and discussed. Finally, Sec. V concludes the paper.

\section{MEASUREMENT MODELS AND Distributions}

Let the true MS location be $\left(x_{0}, y_{0}\right)$. Assume that there are $N$ measurement configurations (MC's) between the MS and the reference BS's. By one $\mathrm{MC}$, we mean to take a measurement of distance from one of three measurement types : TOA (involving one BS), TSOA (involving two BS's), or TDOA (involving two BS's).

For ease of making a simple and unified discussion on some topics to appear later in this paper, here we want to define a function tsgn : $\{$ TOA, TSOA, TDOA $\}$ (denoted as $S_{T}$ for short) $\mapsto\{0,1,-1\}$, whose definition is

$$
\operatorname{tsgn}(\tau)= \begin{cases}0, & \text { if } \tau=T O A \\ 1, & \text { if } \tau=T S O A \\ -1, & \text { if } \tau=T D O A\end{cases}
$$

Let the locations of the two BS's involved in the $i^{\text {th }} \mathrm{MC}$ be denoted as $\left(x_{1, i}, y_{1, i}\right)$ and $\left(x_{2, i}, y_{2, i}\right)$ if the MC is of type TSOA or TDOA. If the $i^{\text {th }} \mathrm{MC}$ comes from TOA, the location of the BS is denoted as $\left(x_{1, i}, y_{1, i}\right)$. The true distance in the $i^{\text {th }} \mathrm{MC}$, whose measurement type is $\tau_{i}\left(\in S_{T}\right)$, is then

$$
\begin{aligned}
t_{i}= & \sqrt{\left(x_{0}-x_{1, i}\right)^{2}+\left(y_{0}-y_{1, i}\right)^{2}}+ \\
& \operatorname{tsgn}\left(\tau_{i}\right) \cdot \sqrt{\left(x_{0}-x_{2, i}\right)^{2}+\left(y_{0}-y_{2, i}\right)^{2}}
\end{aligned},
$$

where the $\left(x_{2, i}, y_{2, i}\right)$ in the case of $\tau_{i}=$ TOA can be arbitrarily assigned (because $\operatorname{tsgn}\left(\tau_{i}\right)=0$, anyway).

The measured distance is then $m_{i}=t_{i}+g_{i}+n_{i}$, where $g_{i}$ is a measurement noise and is modeled as a zero-mean Gaussian random variable with standard deviation $\theta_{i}$, and $n_{i}$ is a NLOS error. Commonly adopted statistic models for $n_{i}$ include Rayleigh, Rician, exponential, and chi-square distributions, etc. The equation on $m_{i}$ can be rewritten as

$$
\xi_{i}=m_{i}-t_{i}=g_{i}+n_{i},
$$

where obviously $\xi_{i}$ represents the overall measurement error. With the assumption that $g_{i}$ and $n_{i}$ are independent, the pdf of $\xi_{i}$ can be 
obtained from the convolution of $g_{i}$ and $n_{i}$ 's pdf's. For two particular cases $\left(n_{i}\right.$ is Rayleigh or exponential), we are able to find close-form expressions for $\xi_{i}$ 's pdf. They are described below.

\section{A. GRS distribution}

In this and the next two subsections, we will drop the index ${ }_{i}$ in $\xi_{i}, g_{i}$, and $n_{i}$, etc. to make notations simpler.

In this subsection, let us consider the case that the NLOS error $n$ is Rayleigh distributed with parameter $\rho$, whose pdf is $f_{n}(n)=\left(n / \rho^{2}\right) \cdot e^{-n^{2} / 2 \rho^{2}} u(n)$, where $u(\cdot)$ is the unit step function. Performing the convolution of $f_{n}(x)$ and the Gaussian measurement noise's pdf $f_{g}(x)$ (assuming $g \sim \operatorname{Gauss}(0, \theta)$, where $\theta$ is the standard deviation), we get the pdf for $\xi$ :

$$
f_{\xi}(x)=\frac{\rho x}{\lambda^{3}} \cdot e^{-x^{2} / 2 \lambda^{2}} \Phi\left(\frac{\rho x}{\theta \lambda}\right)+\frac{\theta}{\sqrt{2 \pi} \lambda^{2}} \cdot e^{-x^{2} / 2 \theta^{2}},
$$

where $\lambda=\sqrt{\rho^{2}+\theta^{2}}$ and $\Phi(\cdot)$ is the cumulative density function (cdf) of the standard Gaussian random variable (r.v.). Equation (4) will be referred to as the $\operatorname{GRS}(\theta, \rho)$ distribution, where GRS stands for Gaussian Rayleigh sum.

In the MLE method, we may have to search $f_{\xi}(x)$ over feasible regions. The search is typically based on gradient vector and Hessian matrix. Therefore, the first and second derivatives of $f_{\xi}(x)$ may be needed. They are listed below :

$$
\begin{gathered}
f_{\xi}^{\prime}(x)=\frac{\rho}{\lambda^{3}}\left(1-\frac{x^{2}}{\lambda^{2}}\right) e^{-x^{2} / 2 \lambda^{2}} \Phi\left(\frac{\rho x}{\theta \lambda}\right)-\frac{\theta x}{\sqrt{2 \pi} \lambda^{4}} \cdot e^{-x^{2} / 2 \theta^{2}}, \\
f_{\xi}^{\prime \prime}(x)=-\frac{\rho x}{\lambda^{5}}\left(3-\frac{x^{2}}{\lambda^{2}}\right) e^{-x^{2} / 2 \lambda^{2}} \Phi\left(\frac{\rho x}{\theta \lambda}\right) \\
+\frac{1}{\sqrt{2 \pi} \lambda^{4}} e^{-x^{2} / 2 \theta^{2}}\left(\frac{\rho^{2}}{\theta}-\theta+\frac{\theta x^{2}}{\lambda^{2}}\right) .
\end{gathered}
$$

\section{B. GES distribution}

In this subsection, The NLOS error $n$ is assumed to be exponentially distributed with parameter $\beta$. In other words, its $\mathrm{pdf}$ is $f_{n}(n)=(1 / \beta) \cdot e^{-x / \beta} \cdot u(n)$. In this case, the overall measurement error $\xi$ is distributed as [5]

$$
f_{\xi}(x)=\frac{1}{\beta} \cdot e^{-\left(\frac{x}{\beta}-\frac{\theta^{2}}{2 \beta^{2}}\right)} \Phi\left(\frac{x}{\theta}-\frac{\theta}{\beta}\right) .
$$

Equation (7) will be referred to as the $\operatorname{GES}(\theta, \beta)$ distribution, where obviously GES stands for Gaussian exponential sum.

Similar to what had been done for the $\operatorname{GRS}(\theta, \rho)$ case, the first and second derivatives of the GES $(\theta, \beta)$ pdf can be computed. They are shown below,

$$
\begin{aligned}
& f_{\xi}^{\prime}(x)=\frac{-1}{\beta}\left(f_{\xi}(x)-\frac{1}{\sqrt{2 \pi} \theta} e^{-x^{2} / 2 \theta^{2}}\right), \\
& f_{\xi}^{\prime \prime}(x)=\frac{-1}{\beta}\left(f_{\xi}^{\prime}(x)+\frac{x}{\sqrt{2 \pi} \theta^{3}} e^{-x^{2} / 2 \theta^{2}}\right) .
\end{aligned}
$$

\section{Gaussian approximation}

Although we obtained Eqs. (4) and (7) for $\xi$ when $n$ is Rayleigh or exponential distributed, it is in general very difficult to find $\xi$ 's pdf in an exact form when $n$ is otherwise distributed. Therefore, when the pdf is needed but too hard to be derived, we may resort to some approximations . Due to its mathematical tractability, Gaussian pdf is what we want to adopt for this approximation in most cases. More specifically speaking, given the mean $\mu$ and standard deviation $\sigma$ of a pdf, it is approximated by $\operatorname{Gauss}(\mu, \sigma)$. For example, $\operatorname{GRS}(\theta, \rho) \approx \operatorname{Gauss}\left(\rho \sqrt{\frac{\pi}{2}}, \sqrt{\theta^{2}+\left(2-\frac{\pi}{2}\right) \rho^{2}}\right)$, and
$\operatorname{GES}(\theta, \beta) \approx \operatorname{Gauss}\left(\beta, \sqrt{\theta^{2}+\beta^{2}}\right)$. By adopting Gaussian approximation, there is an explicit equation for computing the CRLB[7], which serves to evaluate the performances of estimation algorithms. In the case that measurements only involve TOA, the CRLB can be found in the literature (e.g. see [6]). As developed in this paper, however, TOA, TSOA, and TDOA will be treated in a unified fashion to get their CRLB's.

\section{D. $C R L B$}

Let us return to the scenario that the estimation of MS location is based on $N$ MC's, where the $i^{\text {th }}$ measurement type is $\tau_{i}$. Let the variance of the $i^{\text {th }}$ overall measurement error, which has been approximated by a non-zeromean Gaussian r.v., be $\sigma_{i}^{2}$. Let the vector pointing from $\left(x_{1, i}, y_{1, i}\right)$ to $\left(x_{0}, y_{0}\right)$ be denoted as $\Delta P_{1, i}$. It can be written in the polar form as $\left|\Delta P_{1, i}\right| e^{j \phi_{1, i}}$. In a similar way, phase $\phi_{2, i}$ is defined by the vector pointing from the second BS of the $i^{\text {th }}$ MC to the MS. For a reason to be addressed in Sec. III, a sample of a few (say, $K$ ) measurements needs to be made with respect to each MC. With the terms defined above, the Fisher information matrix (FIM) [7] is computed by $I=K \cdot \sum_{i=1}^{N} I_{i}$, where

$$
I_{i}=\left[\begin{array}{ll}
I_{11, i} & I_{12, i} \\
I_{21, i} & I_{22, i}
\end{array}\right] \text {, }
$$

with the matrix elements given by

$$
\begin{gathered}
I_{11, i}=\frac{1}{\sigma_{i}^{2}}\left(\cos \left(\phi_{1, i}\right)+\operatorname{tsgn}\left(\tau_{i}\right) \cdot \cos \left(\phi_{2, i}\right)\right)^{2}, \\
I_{22, i}=\frac{1}{\sigma_{i}^{2}}\left(\sin \left(\phi_{1, i}\right)+\operatorname{tsgn}\left(\tau_{i}\right) \cdot \sin \left(\phi_{2, i}\right)\right)^{2}, \\
I_{12, i}=I_{21, i}=\frac{1}{\sigma_{i}^{2}}\left(\cos \left(\phi_{1, i}\right)+\operatorname{tsgn}\left(\tau_{i}\right) \cdot \cos \left(\phi_{2, i}\right)\right) . \\
\left(\sin \left(\phi_{1, i}\right)+\operatorname{tsgn}\left(\tau_{i}\right) \cdot \sin \left(\phi_{2, i}\right)\right) .
\end{gathered}
$$

Then, the CRLB is equal to the trace of the inverse of $I$. This bound is the smallest mean squared error achievable by any unbiased estimator of MS's position.

\section{IMPROVED MS POSITIONING WITH REDUCED NLOS ERROR}

To improve the precision of the estimated MS location, the NLOS error should be removed (more practically speaking, reduced) if possible. Although the exact value of the NLOS error can not be known (because it is random), its expected value, however, can be found if proper methods are used. In this paper, we propose a moment-based computation to do just that. The key idea is that the parameters in the model of the overall measurement error (i.e. $\xi_{i}$ ) can be computed from sample moments if a sample of a few (say, $K$ ) measurements (for the $i^{\text {th }} \mathrm{MC}$ ) is given. For example, for the GRS case, the parameter estimation for the $i^{\text {th }} \mathrm{MC}$ is done with

$$
\begin{gathered}
\hat{\rho}_{i}=\sqrt[3]{\sqrt{\frac{2}{\pi}} \cdot \frac{1}{\pi-3} \cdot\left(M_{3, i}+2 M_{1, i}^{3}-3 M_{1, i} M_{2, i}\right)}, \\
\hat{\theta}_{i}=\sqrt{M_{2, i}-M_{1, i}^{2}-\left(2-\frac{\pi}{2}\right) \hat{\rho}_{i}^{2}},
\end{gathered}
$$

where $M_{1, i}, M_{2, i}$, and $M_{3, i}$ are the first, second and third sample moments, respectively. For the GES case, the estimation is

$$
\begin{gathered}
\hat{\beta}_{i}=\sqrt[3]{\frac{1}{2} M_{3, i}+M_{1, i}^{3}-\frac{3}{2} M_{1, i} M_{2, i}}, \\
\hat{\theta}_{i}=\sqrt{M_{2, i}-M_{1, i}^{2}-\hat{\beta}_{i}^{2}} .
\end{gathered}
$$


Now that the parameters specifying the statistics of $\xi_{i}$ are obtained, estimation of $\mathrm{E}\left(\xi_{i}\right)$ (expectation of $\xi_{i}$ ), which is denoted as $\hat{\mu}_{i}$, is easy. For example, it is equal to $\sqrt{\pi / 2} \cdot \hat{\rho}_{i}$ in the GRS case, and equal to $\hat{\beta}_{i}$ in the GES case. Similarly, the estimation of the variances of $\xi_{i}$, denoted by $\hat{\sigma}_{i}^{2}$, is easy. It is equal to $\hat{\theta}_{i}^{2}+(2-\pi / 2) \hat{\rho}_{i}^{2}$ for GRS, and $\hat{\theta}_{i}^{2}+\hat{\beta}_{i}^{2}$ for GES.

Rearranging Eq. (3) and taking expectations of the quantities in it, we get $\mathrm{E}\left(t_{i}\right)=\mathrm{E}\left(m_{i}\right)-\mathrm{E}\left(\xi_{i}\right)$, which can be approximated by time averages as $\overline{t_{i}}=M_{1, i}-\hat{\mu}_{i}$. Physically interpreted, $\overline{t_{i}}$ is the distance of the MS to the BS's in the $i^{\text {th }} \mathrm{MC}$, wherein the overestimation due to NLOS error has already been removed (or more precisely speaking, reduced). With $\bar{t}_{i}$ and $\hat{\sigma}_{i}^{2}$ given for $i=1,2, \ldots, N$, the WLSE of $\left(x_{0}, y_{0}\right)$ is computed from the minimization,with respect to $(x, y)$, of $J_{\text {WLSE }}=\sum_{i=1}^{N} J_{i}$, where

$$
\begin{gathered}
J_{i}=\frac{1}{\hat{\sigma}_{i}^{2}}\left(\sqrt{\left(x-x_{1, i}\right)^{2}+\left(y-y_{1, i}\right)^{2}}+\right. \\
\left.\operatorname{tsgn}\left(\tau_{i}\right) \cdot \sqrt{\left(x-x_{2, i}\right)^{2}+\left(y-y_{2, i}\right)^{2}}-\bar{t}_{i}\right)^{2} .
\end{gathered}
$$

On the other hand, if we want to apply the method of MLE, we utilize the joint pdf (jpdf) of measurements as a function of MS's location. Before applying the method of MLE, however, it should be noted that there is an offset from the mode (i.e. the peak) of $\xi_{i}$ 's pdf to the mean. Let us denote this offset as $\delta_{i}$. Then the jpdf to be maximized is $J_{\mathrm{MLE}}=\prod_{i=1}^{N} f_{\xi_{i}}\left(z_{i}\right)$, where

$$
\begin{gathered}
z_{i}=M_{1, i}-\left(\sqrt{\left(x-x_{1, i}\right)^{2}+\left(y-y_{1, i}\right)^{2}}+\right. \\
\left.\operatorname{tsgn}\left(\tau_{i}\right) \cdot \sqrt{\left(x-x_{2, i}\right)^{2}+\left(y-y_{2, i}\right)^{2}}\right)-\delta_{i} .
\end{gathered}
$$

The reason for the occurrence of the $-\delta_{i}$ term in eq. (19) is that $M_{1, i}$ represents the most probable value of the mean instead of the mode, and in MLE, however, we are searching for the mode of a pdf instead of the mean.

In summary, the proposed algorithm for mobile positioning when the type of overall measurement error is known is described below:

1) Make $K$ measurements of distances for each MC, and their first three moments.

2) Calculate relevant pdf parameters by Eqs. (14)-(15) (for GRS) or Eqs. (16)-(17) (for GES).

3) Subtract the expected NLOS error from the average measured distance: $\overline{t_{i}}=M_{1, i}-\sqrt{\pi / 2} \cdot \hat{\rho}_{i}$ for GRS; $\overline{t_{i}}=M_{1, i}-\hat{\beta}_{i}$ for GES.

4) Minimize $J_{\mathrm{WLSE}}$ to get $(\hat{x}, \hat{y})_{\mathrm{WLSE}}$, or maximize $J_{\mathrm{MLE}}$ to get $(\hat{x}, \hat{y})_{\mathrm{MLE}}$.

\section{EXPERIMENTAL RESULTS}

Simulations were conducted to test the performance of the proposed scheme. Experimental results produced by our method are compared to those obtained without NLOS reduction. As an index for performance evaluation, we define a term called estimation efficiency by $\epsilon=\mathrm{CRLB} / \mathrm{MSE}$, where MSE is the mean (or in practice, average) of the squared distance between $\left(x_{0}, y_{0}\right)$ and the estimated MS location $(\hat{x}, \hat{y})$. In the two subsections below, we present the experimental results for the GRS and the GES cases, respectively.

\section{A. The GRS case}

The setup of the MC's are listed in Table I, where the NLOS error is assumed to be Rayleigh (therefore, $\xi_{i} \sim \operatorname{GRS}\left(\theta_{i}, \rho_{i}\right)$ ). According to [5], $\theta_{i}$ was selected to be about $0.1 \mathrm{~km}$, and $\rho_{i}$ was set to be $0.239 \mathrm{~km}$ or so. Five different MS positions, whose coordinates are listed in Table I in the units of kilometers, were tried. In each MC, a sample of 100 measured distances were generated. With these
TABLE I

SETUP OF MC'S FOR GRS

\begin{tabular}{|c|c|c|c|c|c|c|c|}
\hline$i$ & $\tau_{i}$ & $x_{1, i}$ & $y_{1, i}$ & $x_{2, i}$ & $y_{2, i}$ & $\theta_{i}$ & $\rho_{i}$ \\
\hline 1 & TSOA & -6.60 & 4.85 & 7.73 & 4.14 & .1103 & .2431 \\
\hline 2 & TOA & -0.34 & 0.01 & - & - & .0985 & .2359 \\
\hline 3 & TDOA & 0.21 & 7.70 & -6.66 & -3.50 & .0994 & .2375 \\
\hline 4 & TOA & -0.03 & -8.02 & - & - & .1056 & .2465 \\
\hline 5 & TOA & 8.29 & -4.44 & - & - & .1215 & .2413 \\
\hline
\end{tabular}

data and adopting the Gaussian approximation to GRS pdf, we have sufficient information to calculate the CRLB's. They are listed in the second column of Table II.

TABLE II

CRLB AND ESTIMATION EFFICIENCY FOR GRS

\begin{tabular}{|c|c|c|c|c|}
\hline$\left(x_{0}, y_{0}\right)$ & CRLB & $\epsilon_{\text {Conv }}$ & $\epsilon_{\text {WLSE }}$ & $\epsilon_{\text {MLE }}$ \\
\hline$(0,0)$ & .0238 & .0167 & .0939 & .0921 \\
\hline$(6.01,6.15)$ & .0635 & .0034 & .0494 & .0497 \\
\hline$(-5.72,4.40)$ & .0236 & .0015 & .0516 & .0533 \\
\hline$(-6.05,-6.13)$ & .0288 & .0023 & .0394 & .0403 \\
\hline$(4.54,-5.60)$ & .0222 & .0041 & .0576 & .0570 \\
\hline
\end{tabular}

Three methods were applied to estimate $\left(x_{0}, y_{0}\right)$. The first was the conventional WLSE without NLOS error reduction (i.e. replacing the $\bar{t}_{i}$ in Eq. (18) with $M_{1, i}$ ). Its results, expressed in terms of estimation

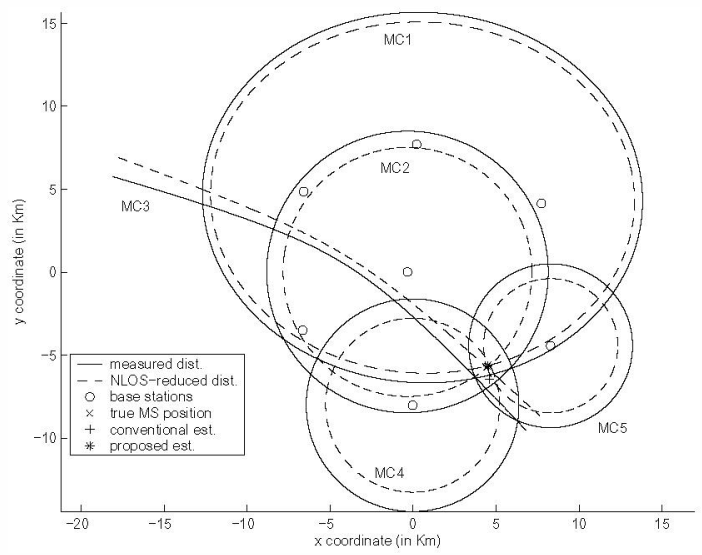

Fig. 1. Trilateration: with and without NLOS error reduction

efficiency, are listed in the third column of Table II. The second method was our proposed WLSE with NLOS error reduction, whose results are listed in the fourth column of Table II. The MLE based on GRS distributions with parameters given by Eqs. (14) and (15) was the third method, whose results constitute the fifth column of Table II.

From Table II, we see that $\epsilon_{\mathrm{WLSE}}$ is much greater than $\epsilon_{\text {Conv }}$ (in the order of tens of times). This shows that MS location estimation is significantly improved by NLOS error reduction. This observation can be explained graphically with Figure 1. In the figure, the solid lines show the average measured distances, which include distance overestimation due to NLOS errors. The dashed lines show the results after the NLOS errors have been reduced. Close inspection shows that the dashed lines corresponding to the various MC's intersect more closely than the solid lines to the true MS position. Also from Table II, we see that $\epsilon_{\mathrm{MLE}}$ is often just slightly greater than $\epsilon_{\mathrm{WLSE}}$ in most cases. In fact, in some rows of Table II, we see that $\epsilon_{\mathrm{MLE}}$ is even a 


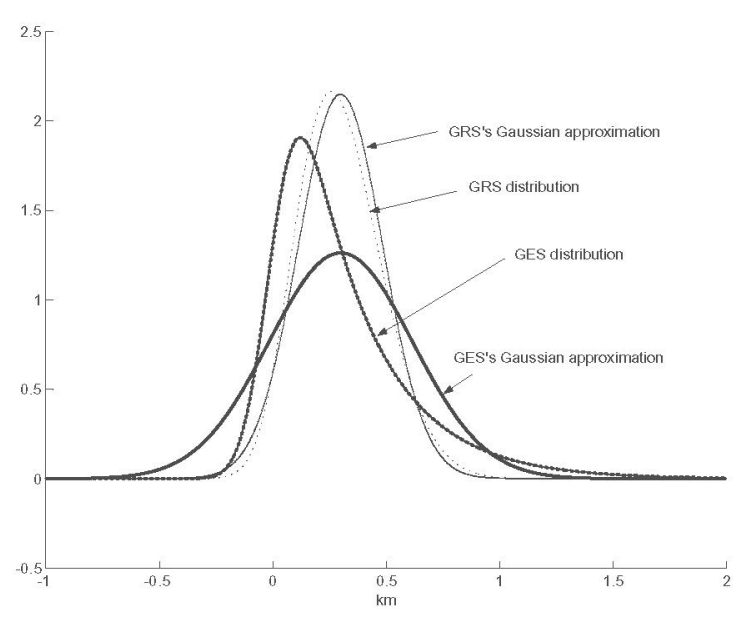

Fig. 2. GRS/GES distribution and Gaussian approximation

little smaller than $\epsilon_{\mathrm{WLSE}}$, probably due to imperfect search for the peak of $J_{\mathrm{MLE}}$. The comparison between $\epsilon_{\mathrm{WLSE}}$ and $\epsilon_{\mathrm{MLE}}$ tells us that WLSE with NLOS error reduction is almost as good as MLE. This can be explained with the help of Figure 2. In the figure, we plot a GRS pdf and its Gaussian approximation. We see that those two curves are extremely close. It means that even if we treat a GRS pdf as a Gaussian pdf, the resulting errors or distortions are negligible. Now that we know the MLE is exactly equal to the WLSE for Gaussian pdf[8], we are not surprised to see the closeness between the MLE and the WLSE for GRS pdf. Realizing that MLE is in general much more involved to compute, we may want to settle for WLSE when the overall measurement error is GRS distributed, as long as the NLOS errors are removed first.

\section{B. The GES case}

The setup of the MC's are listed in Table III, where the NLOS error is assumed to be exponential (therefore, $\xi_{i} \sim \operatorname{GES}\left(\theta_{i}, \beta_{i}\right)$ ). According to [5], $\theta_{i}$ was selected to be about $0.1 \mathrm{~km}$, and $\beta_{i}$ was set to be $0.3 \mathrm{~km}$ or so. Just like the GRS case, a sample of 100 measurements were made for each MC. Simulation results on the estimation efficiencies are displayed in Table IV. Similar to the GRS

TABLE III

SETUP OF MC'S FOR GES

\begin{tabular}{|c|c|c|c|c|c|c|c|}
\hline$i$ & $\tau_{i}$ & $x_{1, i}$ & $y_{1, i}$ & $x_{2, i}$ & $y_{2, i}$ & $\theta_{i}$ & $\beta_{i}$ \\
\hline 1 & TSOA & -6.60 & 4.85 & 7.73 & 4.14 & .1103 & .3120 \\
\hline 2 & TOA & -0.34 & 0.01 & - & - & .0985 & .3521 \\
\hline 3 & TDOA & 0.21 & 7.70 & -6.66 & -3.50 & .0994 & .3021 \\
\hline 4 & TOA & -0.03 & -8.02 & - & - & .1056 & .2987 \\
\hline 5 & TOA & 8.29 & -4.44 & - & - & .1215 & .2858 \\
\hline
\end{tabular}

TABLE IV

CRLB AND ESTIMATION EFFICIENCY FOR GES

\begin{tabular}{|c|c|c|c|c|}
\hline$\left(x_{0}, y_{0}\right)$ & CRLB & $\epsilon_{\text {Conv }}$ & $\epsilon_{\mathrm{WLSE}}$ & $\epsilon_{\mathrm{MLE}}$ \\
\hline$(0,0)$ & .0709 & .0364 & .2743 & .3111 \\
\hline$(6.01,6.15)$ & .1975 & .0092 & .2304 & .2908 \\
\hline$(-5.72,4.40)$ & .0699 & .0041 & .3442 & .4890 \\
\hline$(-6.05,-6.13)$ & .0808 & .0064 & .2151 & .2552 \\
\hline$(4.54,-5.60)$ & .0646 & .0106 & .3522 & .4963 \\
\hline
\end{tabular}

case, here for the GES case we see that $\epsilon_{\mathrm{WLSE}}$ is much greater than $\epsilon_{\text {Conv }}$, which shows the improvement in MS location estimation when NLOS error is reduced. Please notice, however, here in Table IV, $\epsilon_{\mathrm{MLE}}$ is always greater than $\epsilon_{\mathrm{WLSE}}$. Again the reason can be found in Figure 2, wherein a GES pdf and its Gaussian approximation are found. Contrast to the GRS case, we see that GES can not be closely approximated as Gaussian. Therefore, it can not be expected to obtained close results between the WLSE and the MLE parameter estimation for GES pdf. We also know that MLE is in general a more powerful method than WLSE. Therefore, it is reasonable to observe that $\epsilon_{\mathrm{MLE}}$ is greater than $\epsilon_{\mathrm{WLSE}}$ for the GES case.

\section{Comparison between the GRS and GES cases}

In the two subsections above, we compare the values of $\epsilon_{\text {Conv }}, \epsilon_{\mathrm{WLSE}}$ and $\epsilon_{\text {MLE }}$ for the GRS and GES cases separately. In this subsection we want to make a comparison between the two cases as a whole. Comparing Table II and Table IV, we see that the values of $\epsilon_{\text {Conv }}$, $\epsilon_{\mathrm{WLSE}}$ and $\epsilon_{\mathrm{MLE}}$ for GRS are always smaller than the corresponding values for GES. This is because Gaussian distribution is the worst case in all distributions from the viewpoint of randomness. Since GRS is almost Gaussian, as have already been seen in Figure 2, a GRS error is more destructive than a GES error. Therefore, measurements corrupted by GRS errors lead to worse estimation of MS position than those corrupted by GES errors.

\section{CONCLUSIONS}

In this work, we try to improve the accuracy and precision of mobile positioning over conventional WLSE methods. The key idea is to estimate the NLOS error and then subtract it from the measured distance before trilateration is performed. This task is carried out based on the assumption that statistic models for measurement errors are available. In particular, the GRS and GES distributions are considered. Explicit equations for estimating their relevant parameters, based on sample moments, are derived. Measurement types include TOA, TSOA, and TDOA. Regardlessly, they are treated in a unified way. Given a setup of MC's, its corresponding CRLB is derived and is used as a benchmark for performance evaluation. Simulations show that the proposed WLSE with NLOS error reduction performs much better than conventional WLSE. Experimental results also show that WLSE with NLOS error reduction is almost as good as MLE for the GRS case. But for the GES case, MLE outperforms WLSE with NLOS error reduction. The is due to the different degrees of closeness of Gaussian approximation to those two pdf's. One final note is that, with the overall measurement error's model and its associated model parameters being obtained, other ways for estimating MS's location can be feasible (e.g. adopting conditional mean as the estimate).

\section{REFERENCES}

[1] T. S. Rappaport, J. H. Reed, and B. D. Woerner, "Position location using wireless communications on highways of the future," IEEE Communications Magazine, vol. 34, pp. 33-42, Oct. 1996.

[2] H. Koshima and J. Hosen, "Personal locator services emerge," IEEE Spectrum, pp. 41-48, Feb. 2000.

[3] L. Letham, GPS Made Easy: Using Global Positioning Systems in the Outdoors, $4^{\text {th }}$ ed., The Mountaineers Books Co., 2003

[4] J. Caffery Jr. and G. L. Stuber, "Subscriber location in CDMA cellular networks," IEEE Trans. Veh. Technol., vol. 47, pp. 406-416, May 1998.

[5] L. Cong and W. Zhuang, "Non-line-of-sight error mitigation in TDOA mobile location," Proc. IEEE Globecom, pp. 680-684, Nov. 2001.

[6] K. W. Cheung, H. C. So, W. K. Ma, and B. D. Woerner, "Received Signal Strength Based Mobile Positioning via Constrained Weighted Least Squares," Proc. IEEE ICASSP2003, pp. V137-V140, 2003.

[7] R. E. Blahut, Chap. 8 of Principles and Practice of Information Theory, Addison-Wesley Publishing Co., 1987.

[8] Franklin, Powell, and Workman, Chap. 8 of Digital Control of Dynamic Systems, $2^{\text {nd }}$ ed., Addison-Wesley Publishing Co., 1990. 